\title{
A Canonical Structure for Classical Field Theories
}

\author{
Jerzy Kijowski and Wiktor Szczyrba \\ Institute of Mathematical Methods of Physics, \\ Warsaw University, ul. Hoża 74, 00-682 Warsaw, Poland, and \\ Institute of Mathematics of the Polish Academy of Sciences, \\ ul. Śniadeckich 8, 00-956 Warsaw, Poland
}

\begin{abstract}
A general scheme of constructing a canonical structure (i.e. Poisson bracket, canonical fields) in classical field theories is proposed. The theory is manifestly independent of the particular choice of an initial space-like surface in space-time. The connection between dynamics and canonical structure is established. Applications to theories with a gauge and constraints are of special interest. Several physical examples are given.
\end{abstract}

\section{Introduction}

Recent development in the theory of geometrical quantization (cf. $[7,11,13]$ ) has caused a growth of interest in the canonical structure of classical theories. There does not exist however up to now a general canonical formulation of classical field theory. Excepting few simple cases (e.g. the scalar theory $\left(\square+m^{2}\right) \phi=G(\phi)$, cf. $(1,14])$ it is not clear which physical quantities are to be taken as canonical variables and how to define Poisson brackets. Especially difficult are theories with a gauge. It seems that the best way to achieve good results in more complicated cases is "to make a lucky hit" of Poisson brackets. For theories in flat Minkowski space-time the Lorentz invariance is an important guide (cf. [3]). We think, however, that the existence of such fundamental structure as Poisson bracket in a given field theory can not depend on the question if $10 \mathrm{pc}$ away the space-time is curved or not.

In the present paper we are going to formulate a general scheme of the canonical formalism which is consistent with all particular theories known to us. The starting point of our considerations is the finite-dimensional canonical formalism (theory of multisymplectic manifolds) given by one of us $[9]^{1}$. It appears that after a deep reformulation one can apply this approach to a large class of observables (physical quantities, dynamical variables) which contains all physically interesting examples.

\footnotetext{
1 Similar results concerning a finite-dimensional approach to canonical formalism was recently presented by Goldschmidt and Sternberg [17].
} 
The scheme we present here is very general and can be used for very wide class of (non-linear) theories. The price of this generality is that we have had to ignore certain deep mathematical questions connected with non-linear theories (e.g. the geometrical structure of the set of solutions for a given non-linear theory). Most of these problems can only be dealt with in concrete examples. It appears, however, that most of these difficult problems do not interfere with our considerations. We need only some properties of finite-dimensional families of solutions. These properties can be formulated as a set of axioms which are satisfied in theories which have been studied deeply (cf. $[8,12]$ ).

In this way we have been led to the very natural notion of an inductive differential manifold (IDM) which is a generalization of a differential manifold. It seems to us that this notion is much more adequate to study the structure of the set of solutions of field equations than the notion of an infinite dimensional differential manifold. The problem of constructing a differentiable structure in the set of solutions of field equations seems to be extremely difficult in general.

One of the advantages of our theory is the connection established between the canonical structure and the dynamics. Both are defined by the same multisymplectic structure. The Poisson bracket is not a supplement to the field equations but is one of its fundamental structures. The problems (very difficult in general) of covariance of the canonical formalism are automatically solved in our approach. Physical quantities are not functionals in the set of Cauchy data over some space-like surface $\sigma$ (and testing if the Poisson brackets do not depend on $\sigma$ is a rather difficult procedure) but are functionals on the set of complete solutions of the field equations. Our approach gives a considerably simplified treatment of theories with gauge. It appears that physical quantities (for which the Poisson bracket is defined in a natural way) are gauge-invariant functionals. Thus in electrodynamics we do not need to worry about the Poisson brackets for potentials.

In Section 5 we give several examples, but many important observables (e.g. generators of the Poincaré group) have been omitted since they have been examined in [9].

The notation of the present paper is the same as in [9]. In particular, if $\Omega$ is a submanifold of $\mathscr{P}$ embedded by a mapping $i: \Omega \rightarrow \mathscr{P}$ and $\alpha$ is a differential form on $\mathscr{P}$ we denote $\alpha \mid \Omega:=i^{*} \alpha$.

Recently one of us W. Szczyrba, using the general theory elaborated in the present paper has obtained a natural symplectic structure for a set on Einstein metrics in General Relativity. These results will be submitted for publication in Commun. math. Phys.

The authors would like to thank Proffessors K. Maurin and I. Birula-Białynicki for lively interest in their work and fruitful discussions.

We thank also very much Professor D. Simms for his deep comments which were very valuable for us during the preparation of the manuscript.

Our special thanks are due to Professor J. Ehlers for many profound remarks and improvements of the final version of this paper.

\section{Multisymplectic Structure}

The canonical formalism in field theory is usually defined by analogy with mechanics. It appears, that the natural tool is the notion of multi-phase space. 
Definition. By an $n$-phase space we mean a pair $(\mathscr{P}, \gamma)$ where $\mathscr{P}$ is a $r$-dimensional $(r>n)$ differentiable manifold and $\gamma$ is a closed differential $(n+1)$-form on $\mathscr{P}: d \gamma=0$.

The whole dynamics is contained, in this approach, in the form $\gamma$ because of the following:

Definition. A submanifold $\Omega$ embedded in $\mathscr{P}$ is called $\gamma$-singular if for every vector field $X$ tangent to $\mathscr{P}$, defined on $\Omega$ the following is true:

$$
(X\lrcorner \gamma) \mid \Omega=0 .
$$

Definition. By a state in multi-phase space $(\mathscr{P}, \gamma)$ we mean any maximal (i.e. which is not contained in any other) $\gamma$-singular submanifold of $\mathscr{P}$.

In further considerations we shall always assume the regularity condition of $(\mathscr{P}, \gamma)$ (cf. [9]) which assures that each two states are submanifolds of the same dimension.

In mechanics $n=1, \mathscr{P}$ is the $\left(t, q^{i}, p_{j}\right)$-phase space, of Cartan's homogeneous formalism, $\gamma=d\left(\sum p_{j} d q^{j}-H d t\right)$, and states are the usual Hamiltonian trajectories (cf. [9]).

In a field theory Eq. (1.1) are simply field equations and every state is a graph in multi-phase space $\mathscr{P}$ of a solution of the field equations.

As an example take the scalar field theory. We start from the bundle of 4-covectors $\bigwedge^{4} T^{*}(W)$ in the space $W=\mathbb{R} \times M$ where $M$ is space-time. Take in $W$ a coordinate chart $\left(\varphi, x^{\mu}\right)$. There is the canonical 4-form in the bundle $\wedge^{4} T^{*}(W)$

$$
\omega=\eta d x^{0} \wedge d x^{1} \wedge d x^{2} \wedge d x^{3}+\sum_{\mu=0}^{3} \eta^{\mu} d x^{0} \wedge \ldots \wedge d \varphi \wedge \ldots \wedge d x^{3},
$$

where $\left(\varphi, x^{\mu}, \eta, \eta^{\mu}\right)$ is a coordinate chart in $\bigwedge^{4} T^{*}(W)$ given uniquely by the chart $\left(\varphi, x^{\mu}\right)$ in $W$.

As the 4-phase space we take the 9 dimensional submanifold of $\bigwedge^{4} T^{*}(W)$ given by equation

$$
\eta+H\left(\varphi, x^{\mu}, \eta^{\nu}\right)=0 .
$$

The form $\gamma$ is given by: $\gamma=d \omega \mid \mathscr{P}=d(\omega \mid \mathscr{P})$.

It can be easily shown (cf. [9]) that if we parametrize a state by space-time coordinates:

$$
\Omega=\left\{\left(x^{\mu}\right) \rightarrow\left(\varphi\left(x^{\mu}\right), x^{\mu}, \eta^{v}\left(x^{\mu}\right)\right)\right\}
$$

then Eq. (1.1) are equivalent to the field equations:

$$
\begin{aligned}
\partial \varphi / \partial x^{\mu} & =\partial H / \partial \eta^{\mu} \\
\sum_{\mu=0}^{3} \partial \eta^{\mu} / \partial x^{\mu} & =-\partial H / \partial \varphi .
\end{aligned}
$$


Taking e.g. $H=\frac{1}{2}\left(\eta^{\mu} \eta_{\mu}+m^{2} \varphi^{2}\right)-F(\varphi)$ we obtain equations:

$$
\begin{gathered}
\partial \varphi / \partial x^{\mu}=\eta_{\mu}, \\
\left(\square+m^{2}\right) \varphi=G(\varphi)
\end{gathered}
$$

(where $G(\varphi)=F^{\prime}(\varphi)$.

The above approach can be called the multiphase formulation of a field theory. It follows from the geometrical theory of the calculus of variations (see $[5,16])$ that for a given Lagrangian theory there always exists a multiphase formulation of it. A very interesting question is whether this formulation is more general than a Lagrangian one. It is a problem of constructing a multi-phase space for a given system of (partial) differential equations. This problem has already been partially solved and the results will be published soon. In the present paper our starting point is a multisymplectic manifold $(\mathscr{P}, \gamma)$ and we do not need any assumption about its origin.

The set of all states of our theory will be denoted by $\mathscr{H}(\mathscr{P}, \gamma)$ or simply $\mathscr{H}$. We shall assume in the sequel the "hyperbolic" character of $(\mathscr{P}, \gamma)$. This means that there exists in $\mathscr{P}$ a sufficiently large family $\mathscr{C}$ of $(n-1)$-dimensional submanifolds of $\mathscr{P}$ playing the role of "initial data" for the field Eq. (1.2). This has to be understood in the following way: for any $c \subset \mathscr{C}$ there exists a state $\Omega \in \mathscr{H}$ (not necessarily unique) containing $c$ (i.e. $c$ is a submanifold of $\Omega$ ). For the further development of the theory we assume that $\mathscr{C}$ satisfies the set of natural axioms formulated in [9]. In relativistic field theories over space-time $M$ (where $\mathscr{P}$ is a tensor bundle over space-time) the set of all Cauchy data over all space-like surfaces of $M$ satisfies those axioms and can be taken as $\mathscr{C}$.

Elements of the family $\mathscr{C}$ will be called admissible initial surfaces (a.i.s.). Our goal is to define a canonical structure (Poisson brackets) for functionals (of a certain class) defined on the space of states $\mathscr{H}$. Such functionals will be called physical quantities (observables, dynamical variables).

Previously one of us has given a theory of local observables [9]. It appears, however, that there are only very few such quantities (cf. also [17]). A more general approach will be presented in Section 3 .

\section{2. "Pseudo-Differentiable" Structure of the Space of States}

Our main goal in this paper is to find a natural symplectic structure in the space $\mathscr{H}$. It is known that such a structure defines Poisson brackets and the connection between canonical vector fields and physical quantities.

If the set $\mathscr{H}$ was an infinite-dimensional manifold the symplectic structure would be represented by a differential 2 -form $\Gamma$, closed and non-degenerate (in some sense). But a construction of a differentiable structure in $\mathscr{H}$ is in general very difficult. For our purposes it is more natural to use the notion of an "inductive differential manifold" (I.D.M.) which will be defined below.

An inductive differentiable manifold is, roughly speaking, a space in which the notion of finite-dimensional submanifold is defined, and which is "exhausted" by its finite-dimensional submanifolds, in the sense given below. None of these submanifolds, however, may be "thick" enough to fill a neighbourhood of $\Omega$. 
Definition. By an inductive differential manifold of class $C^{s}$ we mean a pair $(\mathscr{H}, \mathscr{A})$ where:

1. $\mathscr{H}$ is a set.

2. $\mathscr{A}$ is a family of injective mappings $\left(\varkappa, P_{k}\right)$ of open $k$-dimensional cubes $P_{k}$ (where $k=0,1,2, \ldots$ ) in $\mathbb{R}^{k}$ :

$$
P_{k}=\left\{\left(t^{1}, t^{2}, \ldots, t^{k}\right):\left|t^{j}\right|<1 \quad j=1,2, \ldots, k\right\}
$$

into $\mathscr{H}$ i.e. $x: P_{k} \rightarrow \mathscr{H}$.

3. The following axioms are satisfied:

a) For every point $\Omega \in \mathscr{H}$ there exists at least one mapping $\left(\varkappa, P_{k}\right) \in \mathscr{A}$ such that $\Omega \in \varkappa\left(P_{k}\right)$.

b) For each two maps $\left(\varkappa_{1}, P_{k_{1}}\right),\left(\varkappa_{2}, P_{k_{2}}\right)$ with intersecting images [i.e. $\varkappa_{1}\left(P_{k_{1}}\right) \cap$ $\varkappa_{2}\left(P_{k_{2}}\right)$ is non-empty] there exists $\left(\varkappa, P_{k}\right)$ such that $x_{1}\left(P_{k_{1}}\right) \subset x\left(P_{k}\right) ; \chi_{2}\left(P_{k_{2}}\right) \subset x\left(P_{k}\right)$.

c) If $\left(\varkappa_{1}, P_{k_{1}}\right),\left(\varkappa_{2}, P_{k_{2}}\right) \in \mathscr{A}$ and $\chi_{1}\left(P_{k_{1}}\right) \subset \varkappa_{2}\left(P_{k_{2}}\right)$ then $x_{2}^{-1} \circ x_{1}: P_{k_{1}} \rightarrow P_{k_{2}}$ is a $C^{\mathrm{s}}$ diffeomorphism onto a submanifold embedded in $P_{k_{2}}$.

d) If $\left(\varkappa, P_{k}\right) \in \mathscr{A}$ and $\varphi: P_{k_{1}} \rightarrow P_{k}$ is a $C^{s}$-diffeomorphism onto a submanifold embedded in $P_{k}$ then $\left(\varkappa^{\circ} \varphi, P_{k_{1}}\right) \in \mathscr{A}$.

The family $\mathscr{A}$ will be called an atlas of $\mathscr{H}$. Its elements will be called finite dimensional (parametrized) surfaces in $\mathscr{H}$.

The axiom d) plays the role of a completness axiom for the atlas $\mathscr{A}$. The family $\mathscr{A}$ defines in $\mathscr{H}$ the inductive topology (cf. [4]).

We shall now define the tangent space at the point $\Omega \in \mathscr{H}$. Consider the family

$$
\mathscr{T}_{\Omega}=\bigcup_{\substack{\left(P_{k}, x\right) \in \mathscr{A} \\ \Omega \in \varkappa\left(P_{k}\right)}}\left(P_{k}, x, e\right),
$$

where " $e$ " is a vector in $\mathbb{R}^{k}$. We introduce in $\mathscr{T}_{\Omega}$ the following equivalence relation: $\left(P_{k_{1}}, \varkappa_{1}, e_{1}\right) \sim\left(P_{k_{2}}, \varkappa_{2}, e_{2}\right)$ if and only if for every surface $\left(\varkappa_{3}, P_{k_{3}}\right)$ containing $\left(\varkappa_{1}, P_{k_{1}}\right)$ and $\left(\varkappa_{2}, P_{k_{2}}\right)[$ in the sense of axiom b)] the following equality holds:

$$
\left(x_{3}^{-1} \circ x_{1}\right)^{\prime}\left(x_{1}^{-1}(\Omega)\right) e_{1}=\left(x_{3}^{-1} \circ x_{2}\right)^{\prime}\left(x_{2}^{-1}(\Omega)\right) e_{2},
$$

where $F^{\prime}(x) \cdot e$ denotes the derivative of the mapping $F$ taken at the point $x$ and acting on the vector $e$.

The quotient space $T_{\Omega}=\mathscr{T}_{\Omega} / \sim$ will be called the tangent space to $\mathscr{H}$ at $\Omega$. It inherits a natural vector space structure from that of the set of representatives:

$$
\alpha\left(P_{k}, x, e\right)+\beta\left(P_{k}, x, f\right)=\left(P_{k}, x, \alpha e+\beta f\right),
$$

where $\alpha, \beta$ are real numbers. The self-consistency of this definition can be easily proved.

For a given inductive differential manifold $\mathscr{H}$ we can define in a natural way such geometrical objects as $C^{s}$-mappings, tangent mapping, vector fields, differential forms, exterior derivative, submanifolds (of finite dimension), Lie derivative etc. (cf. [10]). All these definitions use the possibility of pulling back the corresponding object to $\mathbb{R}^{k}$ using our mappings $\left(\varkappa, P_{k}\right)$. 
For example:

The mapping $\mathscr{H} \ni \Omega \rightarrow \hat{Y}(\Omega) \in T_{\Omega}(\mathscr{H})$ is said to be a $C^{s}$-vector field in $\mathscr{H}$ if for every $\Omega$ there exists a surface $\left(\varkappa, P_{k}\right)$ passing through $\Omega$ [i.e. $\Omega \in \varkappa\left(P_{k}\right)$ ] such that $\hat{Y}$ is a $C^{s}$-vector field tangent to $\chi\left(P_{k}\right)$ in a neighbourhood of $\Omega$ on $\chi\left(P_{k}\right)$.

The last statement is to be understood in the following sense: there exists a vector field (of $C^{s}$-class) $e(x)$ in some neighbourhood of $x^{-1}(\Omega)$ in $P_{k}$ such that $\left(P_{k}, \varkappa, e\left(\varkappa^{-1}(\Omega)\right)\right)$ represents $\hat{Y}(\Omega)$.

As a second example we can take the commutator of two vector fields. We use the fact that "locally" both fields are contained in a common finite-dimensional surface $\left(\chi, P_{k}\right)$. The commutator is thus defined with the use of the commutator of finite-dimensional fields.

Now we shall construct the structure of I.D.M. in the space $\mathscr{H}$ of states of a given multi-phase space $(\mathscr{P}, \gamma)$ by means of families of transformations in $\mathscr{P}$. As we have already noticed (Section 1) there are in general few transformations of $\mathscr{P}$ which carry all states onto states. But for defining local $C^{\mathrm{s}}$-surfaces "in a neighbourhood" of $\Omega$ we need only such transformations which carry $\Omega$ onto states:

Definition. The I.D.M. structure in $\mathscr{H}$ is given by the family $\mathscr{A}$ of all mappings of the following form:

$$
P_{k} \ni\left(t_{1}, \ldots, t_{k}\right) \rightarrow \chi\left(t_{1}, \ldots, t_{k}\right) \in \mathscr{H},
$$

where

$$
\varkappa\left(t_{1}, \ldots, t_{k}\right)=\left\{\psi\left(t_{1}, \ldots, t_{k} ; p\right) \in \mathscr{P}: p \in \Omega, \Omega \in \mathscr{H}\right\}
$$

and $\psi$ is a smooth mapping $P_{k} \times \mathscr{P} \rightarrow \mathscr{P}$ such that

1. For every $\left(t_{1}, \ldots, t_{k}\right) \in P_{k} \psi\left(t_{1}, \ldots, t_{k} ; \cdot\right)$ is a diffeomorphism of $\mathscr{P}$ such that image of $\Omega$ is a state i.e. $\psi\left(t_{1}, \ldots, t_{k} ; \Omega\right) \in \mathscr{H}$.

2. $\psi\left(0_{1}, \ldots, 0 ; \cdot\right)=\mathrm{id}_{\mathscr{P}}$.

3. $\psi$ satisfies the non-degeneracy condition which will be formulated in two stages:

a) If $k=1$ then for every $t \in P_{1}$ there exists $p \in \Omega$ such that the following mapping: $P_{1} \ni t \rightarrow \psi(t ; p) \in \mathscr{P}$ is transversal to $\Omega$ at $(t ; p)$ [i.e. $\left.\partial / \partial t \psi(t ; p) \notin T_{p}(\Omega)\right]$.

b) If $\tau \rightarrow\left(t_{1}(\tau), \ldots, t_{k}(\tau)\right) \in P_{k}$ is a curve in $P_{k}$ with non-vanishing tangent vector then the map $\varphi(\tau ; \cdot)=\psi\left(t_{1}(\tau), \ldots, t_{k}(\tau) ; \cdot\right)$ fulfils the condition a).

The fundamental problem of our theory is to prove that such mappings satisfy Axioms $3 a$ ) and $3 b$ ) of the definition of I.D.M. (the other axioms are satisfied). It is a question about the global geometry of the set of solutions of a given system of field Eq. (1.2). We know, however, that our axiomatics is not empty. It is satisfied by the large class of relativistic, globally hyperbolic field theories. In this case $\mathscr{P}$ is a tensor bundle over space-time $M$, and states are global sections of this bundle. By hyperbolicity we mean a possibility of parametrization of the space $\mathscr{H}$ by the space of Cauchy data over a fixed space-like surface $\sigma \subset M$. Cauchy data are here sections of the bundle $\left(\mathscr{P}_{\sigma}, \pi \mid \sigma, \sigma\right)$ which is the reduction of $(\mathscr{P}, \pi, M)$ to $\sigma \subset M$. Now we can limit ourselves to transformations $\psi \mid \mathscr{P}_{\sigma}$ of the space $\mathscr{P}_{\sigma}$ (and not the whole space $\mathscr{P}$ ). If constraints imposed by the theory on 
the space of initial data are not too complicated (as is the case in all reasonable theories) we can easily prove that the set $\mathscr{A}_{\sigma}$ of mappings generated in $\mathscr{H}$ by families $\psi \mid \mathscr{P}_{\sigma}, \psi \in \mathscr{A}$ satisfies our axioms. Now it remains to prove that different atlases $\mathscr{A}_{\sigma}$ obtained by choosing different space-like surfaces $\sigma$ in $M$ are compatible (i.e. are the same). This is a consequence of global hyperbolicity of our theory.

The I.D.M. approach is even in this case less complicated than a construction of a differentiable structure in $\mathscr{H}$ by using e.g. Cauchy data as local coordinates. In the last case we should have to worry about a topology in the space of Cauchy data, a notion of Fréchet derivative etc. which are very hard problems in the general case. We would like however to mention that our approach can be useful even for theories in which there are no global solutions (because states may have singularities at some points at space-time).

If $\psi$ is such as in 2 a) then

$$
t \rightarrow \Omega_{t}=\{\psi(t ; p) \in \mathscr{P}: p \in \Omega\}=\chi(t)
$$

is a smooth curve in $\mathscr{H}$ such that $\Omega_{0}=\Omega$. Let us take the vector field generated on $\Omega$ by $\psi$ :

$$
\Omega \ni p \rightarrow Y(p)=\left.\frac{d}{d t}\right|_{t=0} \psi(t ; p) \in T_{p}(\mathscr{P}) .
$$

Such vector fields which are defined on $\Omega$ and may be not tangent to $\Omega$ will be called "vector fields on $\Omega$ ". Vector fields on $\Omega$ which are also tangent to $\Omega$ will be called "vector fields in $\Omega$ " (cf. [6]).

Theorem 1. If $\tilde{Y}$ is any prolongation of $Y$ onto a neighbourhood $U$ of $\Omega$ in $\mathscr{P}$ then

$$
\left.\mathscr{L}_{\tilde{Y}}(\tilde{X}\rfloor \gamma\right) \mid \Omega=0
$$

for every vector field $\tilde{X}$ in $U$.

Proof in Section 6.

We would like in the sequel to represent vectors tangent to $\mathscr{H}$ at the point $\Omega$ by vector fields generated by families of diffeomorphisms $\{\psi(t ; \cdot)\}$ by the formula (2.4). We see however that this correspondence can not be univalent. The same curve $t \rightarrow \Omega_{t}$ can be obtained by different diffeomorphisms i.e. the same vector tangent to a given curve can be represented by different vector fields on $\Omega$. Let $\psi_{1}$ and $\psi_{2}$ give the same curve in $\mathscr{H}$ i.e. for every $|t|<1$ the corresponding images are equal:

$$
\psi_{1}(t ; \Omega)=\psi_{2}(t ; \Omega) .
$$

It means that:

$$
\lambda_{t}:=\psi_{2}(t ; \cdot)^{-1} \circ \psi_{1}(t ; \cdot)
$$

transforms $\Omega$ onto $\Omega$ and the vector field $\Omega \ni p \rightarrow d \lambda_{t}(p) / d t$ is tangent to $\Omega$. If we differentiate the formula (2.6) we obtain

$$
d \lambda_{t}(p) /\left.d t\right|_{t=0}=-d \psi_{2} / d t(0 ; p)+d \psi_{1} / d t(0 ; p),
$$


i.e. the difference between vector fields defined on $\Omega$ by $\psi_{1}$ and $\psi_{2}$ is a vector field in $\Omega$ (tangent to $\Omega$ ). Such vector fields satisfy automatically the condition (2.5) since

$$
\left.\left.\mathscr{L}_{\tilde{Y}}(\tilde{X}\lrcorner \gamma\right)\left|\Omega=\mathscr{L}_{\tilde{Y}}\{(\tilde{X}\lrcorner \gamma)\right| \Omega\right\}=0
$$

by virtue of (1.1).

Vector fields in $\Omega$ are generated e.g. by transformations $\psi(t ; \cdot)$ leaving $\Omega$ invariant which correspond to constant curves in $\mathscr{H}\left(\Omega_{t}=\Omega ;|t|<1\right)$, i.e. vector fields in $\Omega$ (and only they) represent the zero vector in $T_{\Omega}(\mathscr{H})$.

Condition (2.7) is not only necessary but is also sufficient for $Y_{1}$ and $Y_{2}$ to be tangent to the same curve $t \rightarrow \Omega_{t}$ : Take $Y_{2}=Y_{1}+X$ where $X$ is tangent to $\Omega$ and $Y_{1}=d \psi_{1} / d t(0 ; \cdot)$. Take any family $\xi_{t}: \mathscr{P} \rightarrow \mathscr{P}$ of diffeomorphisms of $\mathscr{P}$ transforming $\Omega$ onto $\Omega$ "tangently" to $X$ i.e.:

$$
d /\left.d t\right|_{t=0} \xi_{t}(p)=X(p) \text { for } p \in \Omega \text {. }
$$

Then put $\psi_{2}(t ; p):=\psi_{1}\left(t ; \xi_{t}(p)\right)$. Both curves generated by $\psi_{1}$ and $\psi_{2}$ in $\mathscr{H}$ are of course the same and:

$$
d \psi_{2} / d t(0 ; p)=Y_{1}+X=Y_{2}
$$

which was to be proved.

The above considerations show that vectors tangent to $\mathscr{H}$ can be represented by classes of vector fields on $\Omega$ satisfying condition (2.5) modulo vector fields in $\Omega$. Denote the space of such classes by $\tilde{T}_{\Omega}$. In general we do not know if the tangent space $T_{\Omega}(\mathscr{H})$ (which can be identified with a subspace of $\tilde{T}_{\Omega}: T_{\Omega} \subset \tilde{T}_{\Omega}$ ) is equal the whole space $\tilde{T}_{\Omega}$.

A very important role is played in the sequel by the subspace $T_{\Omega}^{0} \subset T_{\Omega}$ composed of vectors corresponding to "spatially compact" deformations of $\Omega$. More precisely: vectors of $T_{\Omega}^{0}$ are represented by such vector fields $Y$ on $\Omega$ that for every a.i.s. $c \subset \Omega$ there is a compact set $K \subset c$ such that $Y$ is tangent to $\Omega$ in $\{c-K\}$.

\section{Symplectic Structure and Poisson Brackets}

We approach now the main point of our considerations: defining in $\mathscr{H}$ a smooth 2-form $\Gamma$. Such a 2-form is a bilinear, antisymmetric functional on the tangent space:

$$
T_{\Omega} \times T_{\Omega} \ni\left(\hat{Y}_{1}, \hat{Y}_{2}\right) \rightarrow \Gamma_{\Omega}\left(Y_{1}, Y_{2}\right) \in \mathbb{R}
$$

According to the general procedure in I.D.M. the smoothness of $\Gamma$ means that $x^{*} \Gamma$ is a smooth 2 -form in $P_{k}$ for every local surface $\left(\varkappa, P_{k}\right) \in \mathscr{A}$. Our 2-form $\Gamma$ will be finite-valued only on a subspace of $T_{\Omega} \times T_{\Omega}$. For the sake of simplicity we shall define it only for such pairs of arguments that at least one of them belongs to $T_{\Omega}^{0}$.

$$
\left.\Gamma_{\Omega}\left(\hat{Y}_{1}, \hat{Y}_{2}\right)=\int_{c \subset \Omega}\left(Y_{1} \wedge Y_{2}\right)\right\lrcorner \gamma,
$$


where $Y_{1}, Y_{2}$ are arbitrary vector fields on $\Omega$ which represent vectors $\hat{Y}_{1}, \hat{Y}_{2}$ (at least one of which belongs to $T_{\Omega}^{0}$ ) and $c \subset \Omega$ is an arbitrary a.i.s. contained in $\Omega$.

Remark 1. If $Y_{1}$ or $Y_{2}$ is tangent to $\Omega$ then $\left.\left(Y_{1} \wedge Y_{2}\right)\right\lrcorner \gamma \mid \Omega=0$ by virtue of the field Eq. (1.1). It follows from the definition of $T_{\Omega}^{0}$ that the integration in the formula (3.1) is extended over a compact set in $c$.

Remark 2. The value of the integral in (3.1) does not depend on the particular choice of field $Y$ representing a vector $\hat{Y}$ by virtue of the same arguments as in Remark 1.

Lemma 1. If the fields $Y_{1}, Y_{2}$ on $\Omega$ satisfy Eq. (2.5) then

$$
\left.d\left[\left(Y_{1} \wedge Y_{2}\right)\right\lrcorner \gamma\right]=0
$$

where $\left(Y_{1} \wedge Y_{2}\right) \perp \gamma$ is understood as a $(n-1)$-form on the manifold $\Omega$.

For the proof see Section 6.

Remark 3. Lemma 1 and the properties of admissible initial surfaces imply that the integral in (3.1) does not depend on the particular choice of $c \in \mathscr{C}$ contained in $\Omega$.

Remark 4. The definition of $\Gamma$ could be extended to a larger class of tangent vectors. Instead of the condition of "spatially bounded" support for fields $Y$ we could impose some weaker condition of "sufficiently rapid vanishing at infinity". Such a condition is necessary if the value of (3.1) has to be finite. For theories with positive energy (as $\lambda \varphi^{4}$ theory with $\lambda<0$ and all physically meaningfull theories) we could reduce the space of states to the space $\mathscr{H}_{\text {fin }}$ which corresponds to finite energy. It seems that the tangent space to $\mathscr{H}_{\text {fin }}$ would belong automatically to the domain of $\Gamma$.

Theorem 2. The form $\Gamma$ is closed, i.e. $d \Gamma=0$.

For proof see the Section 6.

The form $\Gamma$ defines a linear mapping from the tangent to the co-tangent space:

$$
T_{\Omega}^{0} \ni \hat{Y} \rightarrow \hat{Y}^{b}=\Gamma_{\Omega}(\hat{Y}, \cdot) \in T_{\Omega}^{*},
$$

where $T_{\Omega}^{*}$ is the space of linear functionals on $T_{\Omega}$. The image of $T_{\Omega}^{0}$ in $T_{\Omega}^{*}$ will be denoted $T_{\Omega}^{0 \text { b }}$.

Definition. By an observable (physical quantity) we mean any smooth functional $F$ on the space of states $\mathscr{H}$ for which there exists a smooth $T_{\Omega}^{0}$-valued vector field $\Omega \rightarrow \hat{Y}(\Omega) \in T_{\Omega}^{0}$ satisfying

$$
d F=-\hat{Y}^{b} .
$$

Remark 5. It suffices to assume that the field $\hat{Y}$ exists "locally" in the sense of I.D.M. This means that for every point $\Omega \in \mathscr{H}$ there exists a local surface $\left(\varkappa, P_{k}\right)$ passing through $\Omega\left(\Omega \in \varkappa\left(P_{k}\right)\right)$ and the field $\hat{Y}$ tangent to $\chi\left(P_{k}\right)$ in the neighbourhood of $\Omega$, such that (3.3) is satisfied. 
Definition. For two observables $F_{1}$ and $F_{2}$ we put:

$\mathscr{H} \ni \Omega \rightarrow\left\{F_{1}, F_{2}\right\}(\Omega):=\Gamma_{\Omega}\left(\hat{Y}_{1}, \hat{Y}_{2}\right) \in \mathbb{R}$,

where $\hat{Y}_{1}$ and $\hat{Y}_{2}$ are as in (3.3):

$d F_{1}=-\hat{Y}_{1}^{b}$
$d F_{2}=-\hat{Y}_{2}^{b}$.

The functional $\left\{F_{1}, F_{2}\right\}$ is called the Poisson bracket of $F_{1}$ and $F_{2}$.

Remark 6. The definition above is consistent because the right-hand side of (3.4) does not depend on the particular choice of vectors $\hat{Y}_{1}, \hat{Y}_{2}$ satisfying (3.5). To see this take $\hat{Z}_{1}^{b}=\hat{Y}_{1}^{b} ; \hat{Z}_{2}^{b}=\hat{Y}_{2}^{b}$. Then

$$
\begin{aligned}
\Gamma_{\Omega}\left(\hat{Z}_{1}, \hat{Z}_{2}\right) & =\hat{Z}_{1}^{b}\left(\hat{Z}_{2}\right)=\hat{Y}_{1}^{b}\left(\hat{Z}_{2}\right)=\Gamma_{\Omega}\left(\hat{Y}_{1}, \hat{Z}_{2}\right)=-\Gamma_{\Omega}\left(\hat{Z}_{2}, \hat{Y}_{1}\right)=-\hat{Z}_{2}^{b}\left(\hat{Y}_{1}\right) \\
& =-\hat{Y}_{2}^{b}\left(\hat{Y}_{1}\right)=-\Gamma_{\Omega}\left(\hat{Y}_{2}, \hat{Y}_{1}\right)=\Gamma_{\Omega}\left(\hat{Y}_{1}, \hat{Y}_{2}\right) .
\end{aligned}
$$

Theorem 3. The Poisson bracket of two observables is also an observable and $d\left\{F_{1}, F_{2}\right\}=-\left[\hat{Y}_{1}, \hat{Y}_{2}\right]^{b}$,

where $\hat{Y}_{1}, \hat{Y}_{2}$ are as in (3.5).

For the proof see Section 6.

Proposition 1. The set of all observables $\mathscr{F}$ with its natural linear structure and with the form $\{\cdot, \cdot\}$ is a Lie algebra. In particular the Jacobi identity is satisfied:

$\left\{\left\{F_{1}, F_{2}\right\}, F_{3}\right\}+\left\{\left\{F_{2}, F_{3}\right\}, F_{1}\right\}+\left\{\left\{F_{3}, F_{1}\right\}, F_{2}\right\}=0$.

\section{Gauge and Degeneracy}

The mapping $\hat{Y} \rightarrow \hat{Y}^{b}$ is not in general injective, i.e. the form $\Gamma$ is degenerated. The degeneration is connected with:

1. Existence of constraints imposed on the space of Cauchy data (which means that the tangent space $T_{\Omega}$ is small).

2. The fact that an a.i.s. $c$ does not determine uniquely the state $\Omega \supset c$.

In electrodynamics,

1. The initial data $\left\{A_{l}(\boldsymbol{x}), E^{k}(\boldsymbol{x})\right\}$ must satisfy the following constraint

$\operatorname{div} E^{k}=\varrho$,

where $\varrho$ is a charge density.

2. The values of potentials and fields at the time $t_{0}$ do not determine uniquely the values of potentials in the whole space-time.

We deal in this case with the gauge: many states in the geometrical sense correspond to the same physical state (two states which differ only by a gauge transformation are equivalent from the physical point of view). 
However, for hyperbolic theories without constraints the form $\Gamma$ is nondegenerate: the space $T_{\Omega}$ is large enough and the equality

$$
\Gamma_{\Omega}(\hat{Y}, \hat{Z})=0 \quad \text { for every } \quad \hat{Z} \in T_{\Omega}
$$

implies $\hat{Y}=0$. We shall see in the next section that this is the case in the scalar field theory.

In the general case we define the degeneracy distribution:

$$
W_{\Omega}=\left\{\hat{Y} \in T_{\Omega}^{0}: \hat{Y}^{b}=0\right\} .
$$

The distribution $W_{\Omega}$ is involutive:

Proposition 2. If $\hat{Y}_{1}$ and $\hat{Y}_{2}$ are smooth W-valued vector fields in $\mathscr{H}$ then the values of $\left[\hat{Y}_{1}, \hat{Y}_{2}\right]$ lie also in $W$. (For the proof see Section 6.)

Especially interesting are theories for which $W$ is integrable. Then we can divide the space $\mathscr{H}$ into classes composed of integral surfaces of $W$. Two states belong to the same class if they can be joined by a curve the tangent vectors of which belong to $W$. We meet such a situation in electrodynamics: two states which differ by a gradient are put into the same class.

The quotient space $\tilde{\mathscr{H}}$ (space of classes) has the tangent space equal to the quotient

$$
T_{[\Omega]}(\tilde{\mathscr{H}})=T_{\Omega}(\mathscr{H}) / W_{\Omega} .
$$

Using the fact that $\Gamma$ is singular on $W$ we can project $\Gamma$ onto the quotient, obtaining $\tilde{\Gamma}$. The form $\tilde{\Gamma}$ on $\mathscr{\mathscr { H }}$ is closed $(d \tilde{\Gamma}=0)$ as $\Gamma$ is closed on $\mathscr{H}$.

Because $W$ contains the whole degeneracy of $\Gamma$ the quotient form $\tilde{\Gamma}$ is nondegenerate. We thus see that $(\tilde{\Gamma}, \tilde{\mathscr{H}})$ is something like a symplectic I.D.M. which means that the space of physical states has a symplectic structure.

If there is no gauge in our theory then $\tilde{\mathscr{H}}=\mathscr{H}$ and $(\mathscr{H}, \Gamma)$ is already symplectic. to $W$ :

Let us notice now that the forms $\hat{Y}^{b}$ (where $\hat{Y} \in T_{\Omega}^{0}$ ) vanish on vectors belonging

$$
\left\langle\hat{X}, \hat{Y}^{b}\right\rangle=\Gamma_{\Omega}(\hat{Y}, \hat{X})=-\Gamma_{\Omega}(\hat{X}, \hat{Y})=-\left\langle\hat{Y}, \hat{X}^{b}\right\rangle=0
$$

for $\hat{X} \in W_{\Omega}$.

We thus see that the condition (3.3) means that $d F$ vanishes on vectors tangent to the gauge-congruence (in the case where $W$ is integrable), i.e. observables are gauge-invariant functionals, so they can be treated as functionals on $\tilde{\mathscr{H}}$. The whole canonical structure is thus reduced to the symplectic geometry in $(\tilde{\mathscr{H}}, \tilde{\Gamma})$, and our definition of Poisson bracket coincides with the usual definition in the symplectic case.

Every observable defines the unique vector field $\left[\hat{Y}_{F}\right]$ on $\tilde{\mathscr{H}}$ (which is a class of vector fields in $\mathscr{H}$ modulo fields tangent to the gauge congruence) by the formula (3.3). The uniqueness follows from the fact that $\hat{Y}_{F}$ is determined in $\mathscr{H}$ up to vector fields belonging to $W$ (tangent to the gauge). Such fields in $\tilde{\mathscr{H}}$ will be called canonical. 
Theorem 4.1. Canonical fields with Lie bracket $[\cdot, \cdot]$, form a Lie algebra and the mapping $\mathscr{F} \ni F \rightarrow\left[\hat{Y}_{F}\right]$ is a homomorphism of Lie algebras.

2. The kernel of this mapping is composed of constant functionals.

1. Is an immediate consequence of Theorem 3.2. Is a consequence of the nondegeneracy of $\tilde{\Gamma}$.

\section{Examples}

As a first example we shall consider a non-linear electrodynamics (cf. $[9,16]$ ) and compute equal-time Poisson brackets for components $B^{k}$ and $D^{k}$ of magnetic and electric fields.

By $M$ we denote now Minkowski's space-time with metric tensor $(+,-,-,-)$. Take the space $W=T^{*}(M)$ as a configuration space. We have in $W$ local coordinates $\left(x^{\mu}, A_{\mu}\right)$ induced by local coordinates $\left(x^{\mu}\right)$ in $M$. The phase space

$\mathscr{P} \subset \stackrel{4}{\wedge} T^{*}(W)$ is composed of 4-covectors in $W$ which have the form:

$$
h d x^{0} \wedge \ldots \wedge d x^{3}+\sum h^{\mu v} d x^{0} \wedge \ldots \wedge d A_{\hat{v}} \wedge \ldots \wedge d x^{3}
$$

and $h^{\mu \nu}=-h^{\nu \mu} ; h=H\left(x^{\mu}, A_{\mu}, h^{\mu \nu}\right)$.

For the sake of simplicity of notation consider the case when currents vanish, i.e. $H=H\left(h^{\mu v}\right)$. In this situation the canonical $(4+1)$-form in $\mathscr{P}$ is

$$
\gamma=(1 / 2) \partial H / \partial h^{\mu v} d h^{\mu v} \wedge d x^{0} \wedge \ldots \wedge d x^{3}+d h^{\mu v} \wedge d x^{0} \wedge \ldots \wedge d d_{\hat{v}} \wedge \ldots \wedge d x^{3}
$$

(the summation convention is used). The equations of motion $(1.2)$ are (cf. $[9,16])$

$$
\begin{aligned}
f_{\mu \nu}: & =\partial H / \partial h^{\mu \nu}=\partial_{\mu} A_{\nu}-\partial_{\nu} A_{\mu} \\
\partial_{\nu} h^{\mu \nu} & =0
\end{aligned}
$$

The space $\mathscr{P}$ has bundle structure over $M(\pi: \mathscr{P} \rightarrow M)$. Solutions of field Eq. (5.2) are sections of this bundle, given in local coordinates $\left(x^{\mu}, A_{\mu}, h^{\mu v}\right)$ by functions

$$
\begin{aligned}
A_{\mu} & =A_{\mu}\left(x^{\nu}\right) \\
h^{\mu \nu} & =h^{\mu \nu}\left(x^{\nu}\right)
\end{aligned}
$$

satisfying (5.2). The set $\mathscr{C}$ of a.i.s. consists of all 3-dimensional submanifolds which are sections of bundles $\mathscr{P}_{\sigma}$ (where $\mathscr{P}_{\sigma}$ is a reduction of $\mathscr{P}$ to an arbitrary spacelike surface $\sigma$ ) and which satisfy constraints analogous to (4.1). For the sake of simplicity take $\sigma=\left\{x \in M ; x^{0}=\right.$ const $\}$. In this case the constraints are

$$
\begin{aligned}
\partial_{k} h^{0 k} & =0 \\
f_{k l} & =\partial_{k} A_{l}-\partial_{l} A_{k}
\end{aligned}
$$

where we suppose that $f_{k l}$ are functions of $h^{\mu \nu}$. 
Let $\hat{Y}$ be a vector tangent to $\mathscr{H}$ at $\Omega$. It can be represented by a $\pi$-vertical vector field $Y$ defined on $\Omega$. In local coordinates:

$$
Y=Q_{\mu} \partial / \partial A_{\mu}+\sum_{\mu<v} P^{\mu \nu} \partial / \partial h^{\mu \nu}
$$

It is easy to check that $Y$ satisfies Eq. (2.5) only if

$$
\begin{aligned}
\partial_{\mu} Q_{v}\left(x^{\lambda}\right)-\partial_{v} Q_{\mu}\left(x^{\lambda}\right) & =1 / 2 \partial f_{\mu v} / \partial h^{\alpha \beta} P^{\alpha \beta} \\
\partial_{v} P^{\mu \nu}\left(x^{\lambda}\right) & =0 \\
P^{\mu v} & =-P^{v \mu} .
\end{aligned}
$$

If $\hat{Y}_{1}$ and $\hat{Y}_{2}$ are given by $\left(Q_{\mu}, P_{1}^{\mu \nu}\right)$ and $\left(\frac{Q_{\mu}}{P_{2}}{\underset{2}{\mu \nu}}^{\mu \nu}\right.$ according to (5.5) then

$$
\left.\Gamma_{\Omega}\left(\hat{Y}_{1}, \hat{Y}_{2}\right)=\int_{c}\left(Y_{1} \wedge Y_{2}\right)\right\lrcorner \gamma=\int_{\sigma}\left(\begin{array}{c}
P^{k 0} \\
1 \\
2
\end{array} \underset{2}{Q_{k}}-\underset{1}{P^{k 0}} Q_{k}\right) d x^{1} \wedge d x^{2} \wedge d x^{3}
$$

Vectors from the subspace $T_{\Omega}^{0}$ are represented by vector fields such that corresponding functions $Q_{\mu}$ and $P^{\mu v}$ have spatially compact supports. Let us define the following observable-valued distributions:

$$
\begin{aligned}
& C_{0}^{\infty}(\sigma) \ni \psi \rightarrow D^{k}(\psi) \in \mathscr{F} \\
& C_{0}^{\infty}(\sigma) \ni \psi \rightarrow B^{k}(\psi) \in \mathscr{F},
\end{aligned}
$$

where

$$
\begin{aligned}
& D^{k}(\psi)=\int_{\sigma} h^{k 0} \cdot \psi d x^{1} \wedge d x^{2} \wedge d x^{3} \\
& B^{k}(\psi)=-1 / 2 \varepsilon^{k i j} \int_{\sigma} f_{i j} \cdot \psi d x^{1} \wedge d x^{2} \wedge d x^{3} .
\end{aligned}
$$

The value of $D^{k}(\psi)$ and $B^{k}(\psi)$ is thus the value of $k$-th component of electric (magnetic) field smeared with the test function $\psi$.

Take any vector $\hat{Z}$ tangent to $\mathscr{H}$ and its representative

$$
Z=U_{\mu} \partial / \partial A_{\mu}+V^{\mu v} \partial / \partial h^{\mu \nu}
$$

where $U_{\mu}$ and $V^{\mu v}$ satisfy Eq. (5.6).

The infinitesimal change of the value of $D^{k}(\psi)$ and $B^{k}(\psi)$ caused by the change of a state connected with the displacement of initial data (5.4) along the field $Z$ is given by the formula:

$$
\begin{aligned}
& d D^{k}(\psi) \hat{Z}=\int_{\sigma} V^{k 0} \cdot \psi d x^{1} \wedge d x^{2} \wedge d x^{3}, \\
& d B^{k}(\psi) \hat{Z}=-1 / 2 \varepsilon^{k i j} \int_{\sigma}\left(\partial_{i} U_{j}-\partial_{j} U_{i}\right) \psi d x^{1} \wedge d x^{2} \wedge d x^{3} .
\end{aligned}
$$

If $Y_{D^{k}}$ is such that

$$
Q_{j} \mid \sigma=\delta_{j}^{k} \psi \quad \text { and } \quad P^{i 0} \mid \sigma=0
$$


then comparing (5.10) with (5.7) we see that

$$
d D^{k}(\psi) \hat{Z}=-\Gamma_{\Omega}\left(\hat{Y}_{D^{k}}, \hat{Z}\right)=\left\langle\hat{Z},-\hat{Y}_{D^{k}}^{b}\right\rangle
$$

i.e. $d D^{k}(\psi)=-\hat{Y}_{D^{k}}^{b}$.

Integrating (5.11) by parts we obtain

$$
d B^{k}(\psi) \hat{Z}=-\int_{\sigma} \varepsilon^{k i j} U_{i} \partial_{j} \psi d x^{1} \wedge d x^{2} \wedge d x^{3} .
$$

Take $Y_{B^{k}}$ such that

$$
Q_{j}\left|\sigma=0, \quad P^{i 0}\right| \sigma=\varepsilon^{i j k} \partial_{j} \psi .
$$

Comparing (5.14) with (5.7) we obtain

$$
d B^{k}(\psi) \hat{Z}=-\Gamma_{\Omega}\left(\hat{Y}_{B^{k}}, \hat{Z}\right)=\left\langle\hat{Z},-\hat{Y}_{B^{k}}^{b}\right\rangle .
$$

Hence $d B^{k}(\psi)=-\hat{Y}_{B^{k}}^{b}$.

The Poisson brackets are:

$$
\begin{aligned}
\left\{B^{k}\left(\psi_{1}\right), B^{s}\left(\psi_{2}\right)\right\} & =\Gamma\left(\hat{Y}_{B^{k}}\left(\psi_{1}\right), \hat{Y}_{B^{s}}\left(\psi_{2}\right)\right)=0, \\
\left\{D^{k}\left(\psi_{1}\right), D^{s}\left(\psi_{2}\right)\right\} & =\Gamma\left(\hat{Y}_{D^{k}}\left(\psi_{1}\right), \hat{Y}_{D^{s}}\left(\psi_{2}\right)\right)=0, \\
\left\{B^{k}\left(\psi_{1}\right), D^{s}\left(\psi_{2}\right)\right\} & =\Gamma\left(\hat{Y}_{B^{k}}\left(\psi_{1}\right), \hat{Y}_{D^{s}}\left(\psi_{2}\right)\right) \\
& =\varepsilon^{s j k} \int\left(\partial_{j} \psi_{1}\right) \psi_{2} d x^{1} \wedge d x^{2} \wedge d x^{3} .
\end{aligned}
$$

If we strip the above formulae of test functions $\psi_{1}$ and $\psi_{2}$ we obtain the Poisson brackets in the distribution form:

$$
\begin{aligned}
& \left\{B^{k}(\boldsymbol{x}), B^{s}(\boldsymbol{y})\right\}=0=\left\{D^{k}(\boldsymbol{x}), D^{s}(\boldsymbol{y})\right\} \\
& \left\{B^{k}(\boldsymbol{x}), D^{s}(\boldsymbol{y})\right\}=-\varepsilon^{k s j} \partial_{j} \delta(\boldsymbol{x}-\boldsymbol{y}) .
\end{aligned}
$$

As a second example we shall compute the commutation relations for the components of the energy-momentum tensor in the non-linear Klein-Gordon theory. Let $M$ be space-time and $W=M \times \mathbb{R}$. Let $\left(x^{\mu}\right)$ denote local coordinates on $M$ and $\left(x^{\mu}, \varphi\right)$ local coordinates on $W$. The phase space $\mathscr{P} \subset \bigwedge^{4} T^{*}(W)$ is composed of 4-forms given by formula (1.4) which satisfy the condition (1.5). We take

$$
H\left(\varphi, \eta^{\mu}\right)=1 / 2 \eta^{\mu} \eta_{\mu}+G(\varphi) \quad \text { where } \quad G \in C^{\infty}(\mathbb{R}) .
$$

There is on $\mathscr{P}$ the canonical 5-form $\gamma=d \omega \mid \mathscr{P}$ where

$$
\begin{aligned}
\omega\left(x^{\mu}, \varphi, \eta^{\nu}\right)= & -H\left(\varphi, \eta^{\mu}\right) d x^{0} \wedge d x^{1} \wedge d x^{2} \wedge d x^{3} \\
& +\eta^{\mu} d x^{0} \wedge \ldots \wedge d \varphi \wedge \ldots \wedge d x^{3} .
\end{aligned}
$$


Because $H$ does not depend on $\left(x^{\mu}\right)_{\mu=0}^{3}$ space-time translations in $\mathscr{P}$, i.e. fields $X=a^{\mu} \partial / \partial x^{\mu}$ (where $a^{\mu}$ are constant) preserve $\gamma$ :

$$
\left.\left.\mathscr{L}_{X} \omega=0=d(X\lrcorner \omega\right)+X\right\lrcorner \gamma .
$$

Such fields $X$ are thus local canonical fields and 3-forms $\left.\partial / \partial x^{\mu}\right\rfloor \omega$ determine the energy-momentum tensor (cf. [9]).

States of this theory are sections of the bundle $\pi: \mathscr{P} \rightarrow M$. In local coordinates they are given by functions:

$$
\varphi=\varphi\left(x^{\mu}\right), \quad \eta^{\mu}=\eta^{\mu}\left(x^{\mu}\right)
$$

where

$$
\begin{aligned}
\square \varphi+G^{\prime}(\varphi) & =0 \\
\eta^{\mu} & =g^{\mu \nu} \partial \varphi / \partial x^{\nu}
\end{aligned}
$$

where $g^{\mu v}$ is the metric tensor.

The set $\mathscr{C}$ of all a.i.s. is equal to sections of all reductions $\mathscr{P} \mid \sigma$ of $\mathscr{P}$ to spacelike surfaces $\sigma \subset M$ satisfying some compatibility conditions implied by (5.25). For $\sigma=\left\{x^{\circ}=\right.$ const $\}$ this condition is following

$$
-\eta^{k}=\eta_{k}=\partial \varphi / \partial x^{k} \quad k=1,2,3 .
$$

For any state $\Omega$ given by (5.24), (5.25) a $\pi$-vertical vector field on $\Omega Y=Q \partial / \partial \varphi+$ $P^{\mu} \partial / \partial \eta^{\mu}$ can represent a tangent vector to $\mathscr{H}$ at $\Omega$ only if

$$
\begin{aligned}
\square Q+G^{\prime \prime}(\varphi) Q & =0 \\
P^{\mu} & =g^{\mu \nu} \partial_{\nu} Q .
\end{aligned}
$$

Let $c$ be an a.i.s. generated by the space-like surface $\sigma=\left\{\left(x^{\mu}\right): x^{0}=\right.$ const $\}$. If the fields $Y_{1}, Y_{2}$ on $\Omega$ fulfil (5.26) and at least one of them has a compact support on $c$ then using formulae (3.1) and (5.21) we obtain:

$$
\Gamma_{\Omega}\left(\hat{Y}_{1}, \hat{Y}_{2}\right)=\int_{\sigma}\left(P_{1}^{0} Q_{2}-P_{2}^{0} Q_{1}\right) d x^{1} \wedge d x^{2} \wedge d x^{3}
$$

We see from (5.27) that $\Gamma$ is here non-degenerate. Values of $Q$ and $P^{0}$ on $\sigma$ can be taken completely arbitrarily. If thus (5.27) vanishes for every $Q_{1}$ and $P_{1}^{0}$ then $Q_{2}=0$ and $P_{2}^{0}=0$ on $\sigma$. But Eq. (5.26) imply that $Q_{2}=0, P_{2}=0$ on $\Omega$ i.e. $\hat{Y}_{2}=0$.

The energy-momentum tensor is

$$
T^{\mu v}=\eta^{v} \eta^{\mu}-g^{v \mu}\left(1 / 2 \eta^{\lambda} \eta_{\lambda}-G(\varphi)\right)
$$

according to the following formula:

$$
\left.\left(\partial / \partial x^{\mu}\right\rfloor \omega\right) \mid \Omega=\sum_{\nu=0}^{3}(-1)^{v+1}\left(\eta^{v} \eta_{\mu}-\delta_{\mu}^{v}\left(1 / 2 \eta^{\lambda} \eta_{\lambda}-G(\varphi)\right)\right) d x^{0} \wedge \ldots \ldots \wedge d x^{3} .
$$


We define observable-valued distributions

$$
C^{\infty}(\sigma) \ni \psi \rightarrow T^{\mu \nu}(\psi)=\int_{\sigma} T^{\mu v}(0, \boldsymbol{x}) \psi(\boldsymbol{x}) d x^{1} \wedge d x^{2} \wedge d x^{3}
$$

If $Z=U \partial / \partial \varphi+V^{\mu} \partial / \partial \eta^{\mu}$ is any $\pi$-vertical vector field on $\Omega$ fulfilling (5.26) then according to (5.28) we have:

$$
d T^{00}(\psi) \hat{Z}=\int_{\sigma}\left(\eta_{0} V^{0}-\eta_{k} V^{k}+G^{\prime}(\varphi) U\right) \psi d x^{1} \wedge d x^{2} \wedge d x^{3} .
$$

Using (5.25), (5.26) and integrating by parts we obtain

$$
d T^{00}(\psi) \hat{Z}=\int_{\sigma}\left(\eta_{0} V^{0} \psi-U\left(\psi \partial_{0} \eta^{0}-\eta^{k} \partial_{k} \psi\right)\right) d x^{1} \wedge d x^{2} \wedge d x^{3} .
$$

If $Y^{00}$ is given by $\left(Q, P^{\mu}\right)$ such that

$$
Q \mid \sigma=\eta_{0} \psi \quad \text { and } \quad P^{0} \mid \sigma=\psi \partial_{0} \eta^{0}-\eta^{k} \partial_{k} \psi
$$

then comparing (5.32) with (5.27) we have:

$$
d T^{00}(\psi) \hat{Z}=\left\langle\hat{Z},-\left(\hat{Y}^{00}\right)^{b}\right\rangle .
$$

In the similiar way we compute:

$$
d T^{0 k}(\psi) \hat{Z}=\int_{\sigma}\left(V^{0} \eta_{k}+V^{k} \eta_{0}\right) \psi d x^{1} \wedge d x^{2} \wedge d x^{3} .
$$

Using (5.25) and (5.26) we obtain

$$
d T^{0 k}(\psi) \hat{Z}=\int_{\sigma}\left(-V^{0} \eta_{k} \psi+U \cdot \partial_{k}\left(\eta_{0} \psi\right)\right) d x^{1} \wedge d x^{2} \wedge d x^{3} .
$$

If $Y^{0 k}$ is given by $\left(Q, P^{\mu}\right)$ such that

$$
Q \mid \sigma=-\eta_{k} \psi \quad \text { and } \quad P^{0} \mid \sigma=-\partial_{k}\left(\eta_{0} \psi\right)
$$

then we have

$$
d T^{0 k}(\psi) \hat{Z}=\left\langle Z,-\left(\hat{Y}^{0 k}\right)^{b}\right\rangle .
$$

Now

$$
d T^{k l}(\psi) \hat{Z}=\int_{\sigma}\left(-V^{k} \eta^{l}-V^{l} \eta^{k}-g^{k l}\left(\eta_{0} V^{0}+\eta_{s} V^{s}-G^{\prime}(\varphi) U\right)\right) \psi d x^{1} \wedge d x^{2} \wedge d x^{3} .
$$

Using again (5.25), (5.26) and integrating by parts we obtain

$$
\begin{aligned}
d T^{k l}(\psi) \hat{Z}= & \int_{\sigma} U\left(-\partial_{k} \eta^{l} \cdot \psi-\partial_{l} \eta^{k} \cdot \psi-\eta^{l} \cdot \partial_{k} \psi-\eta^{k} \partial_{l} \psi+g^{k l}\left(\partial_{0} \eta^{0} \cdot \psi-\eta^{s} \partial_{s} \psi\right)\right. \\
& \left.+g^{k l} V^{0} \eta_{0} \psi\right) d x^{1} \wedge d x^{2} \wedge d x^{3}
\end{aligned}
$$

If $Y^{k l}$ is given by $\left(Q, P^{\mu}\right)$ such that

$$
Q \mid \sigma=g^{k l} \eta_{0} \psi
$$


and

$$
P^{0} \mid \sigma=\partial_{k}\left(\eta^{l} \psi\right)+\partial_{l}\left(\eta^{k} \psi\right)+g^{k l}\left(\eta^{s} \partial_{s} \psi-\partial_{0} \eta^{0} \psi\right)
$$

we have

$$
d T^{k l}(\psi) \hat{Z}=\left\langle\hat{Z},-\left(\hat{Y}^{k l}\right)^{b}\right\rangle .
$$

Now we can compute the Poisson brackets

$$
\begin{aligned}
\left\{T^{0 k}\left(\psi_{1}\right), T^{0 l}\left(\psi_{2}\right)\right\} & =\Gamma\left(\hat{Y}^{0 k}\left(\psi_{1}\right), \hat{Y}^{0 l}\left(\psi_{2}\right)\right) \\
& =\int_{\sigma}\left(\eta_{0} \eta_{k} \psi_{2} \partial_{l} \psi_{1}-\eta_{0} \eta_{l} \psi_{1} \partial_{k} \psi_{2}\right) d x^{1} \wedge d x^{2} \wedge d x^{3} \\
& =\int_{\sigma}\left(T^{0 l} \psi_{1} \partial_{k} \psi_{2}-T^{0 k} \psi_{2} \partial_{l} \psi_{1}\right) d x^{1} \wedge d x^{2} \wedge d x^{3} .
\end{aligned}
$$

In distribution notation we write

$$
\begin{aligned}
& \left\{T^{0 k}(\boldsymbol{x}), T^{0 l}(\boldsymbol{y})\right\}=T^{0 l}(\boldsymbol{x}) \partial_{k} \delta(\boldsymbol{x}-\boldsymbol{y})+T^{0 k}(\boldsymbol{y}) \partial_{l} \delta(\boldsymbol{x}-\boldsymbol{y}) \\
& \begin{aligned}
\left\{T^{00}\left(\psi_{1}\right), T^{00}\left(\psi_{2}\right)\right\} & =\Gamma\left(\hat{Y}^{00}\left(\psi_{1}\right), \hat{Y}^{00}\left(\psi_{2}\right)\right) \\
& =\int_{\sigma}\left(\eta^{k} \eta_{0} \psi_{1} \partial_{k} \psi_{2}-\eta^{k} \eta_{0} \psi_{2} \partial_{k} \psi_{1}\right) d x^{1} \wedge d x^{2} \wedge d x^{3} \\
& =\int_{\sigma}\left(T^{k 0} \psi_{1} \partial_{k} \psi_{2}-T^{k 0} \psi_{2} \partial_{k} \psi_{1}\right) d x^{1} \wedge d x^{2} \wedge d x^{3} .
\end{aligned}
\end{aligned}
$$

In distribution notation:

$$
\left\{T^{00}(\boldsymbol{x}), T^{00}(\boldsymbol{y})\right\}=T^{k 0}(\boldsymbol{x}) \partial_{k} \delta(\boldsymbol{x}-\boldsymbol{y})+T^{k 0}(\boldsymbol{y}) \partial_{k} \delta(\boldsymbol{x}-\boldsymbol{y}) .
$$

In a similiar way:

$$
\begin{aligned}
& \left\{T^{00}(\boldsymbol{x}), T^{0 k}(\boldsymbol{y})\right\}=T^{k s}(\boldsymbol{x}) \partial_{s} \delta(\boldsymbol{x}-\boldsymbol{y})+T^{00}(\boldsymbol{y}) \partial_{k} \delta(\boldsymbol{x}-\boldsymbol{y}), \\
& \left\{T^{k l}(\boldsymbol{x}), T^{00}(\boldsymbol{y})\right\} \\
& \quad=\partial_{0} T^{k l}(\boldsymbol{x}) \delta(\boldsymbol{x}-\boldsymbol{y})+T^{l 0}(\boldsymbol{x}) \partial_{k} \delta(\boldsymbol{x}-\boldsymbol{y})+T^{k 0}(\boldsymbol{x}) \partial_{l} \delta(\boldsymbol{x}-\boldsymbol{y}) .
\end{aligned}
$$

The same formulae can be obtained for a non-linear electrodynamics with vanishing currents (cf. [2]).

\section{Proofs}

In this section we shall use the following notation. If $\Omega$ is a submanifold embedded in $\mathscr{P}$ and $X, Y, Z$ are vector fields on $\Omega$ (tangent to $\mathscr{P}$ and defined on $\Omega$ ) then we shall denote by $\tilde{X}, \tilde{Y}, \tilde{Z}$ any extensions of $X, Y, Z$ onto some neighbourhood of $\Omega$ in $\mathscr{P}$. If $\stackrel{p}{\omega}$ is any $p$-form on $\mathscr{P}$ and $X_{0}, X_{1}, \ldots, X_{p-1}, X_{p}$ are vector fields in $\mathscr{P}$ 
then we shall use the following formulae (cf. [15]):

$$
\begin{aligned}
& \left.p \cdot \stackrel{p}{\omega}\left(X_{0}, X_{1}, \ldots, X_{p-1}\right)=\left(X_{0}\right\rfloor \stackrel{p}{\omega}\right)\left(X_{1}, \ldots, X_{p-1}\right) \\
& =1 /(p-1) !\left\langle X_{0} \wedge X_{1} \wedge \ldots \wedge X_{p-1} \mid \omega\right\rangle \\
& (p+1) d \stackrel{p}{\omega}\left(X_{0}, \ldots, X_{p}\right)=\sum_{k=0}^{p}(-1)^{k} X_{k}\left(\stackrel{p}{\omega}\left(X_{0}, \ldots, ., X_{p}\right)\right) \\
& +\sum_{0 \leqq k<s \leqq p}(-1)^{k+s} \stackrel{p}{\omega}\left(\left[X_{k}, X_{s}\right], X_{0}, \ldots \ldots \ldots, X_{p}\right), \\
& \left.\mathscr{L}_{X_{k}} \stackrel{p}{\omega}=d\left(X_{k}-\stackrel{p}{\omega}\right)+X_{k}\right\rfloor d \stackrel{p}{\omega}, \\
& \mathscr{L}_{X_{k}}\left(X_{j}\right)=\left[X_{k}, X_{j}\right] \text {. }
\end{aligned}
$$

Proof of Theorem 1. If $\psi$ was the local group of diffeomorphisms generated by $Y$ (at least in a neighbourhood $[-\varepsilon, \varepsilon] \times U$ of the set $\{0\} \times \Omega \subset \mathbb{R} \times \mathscr{P}$ ) then (2.5) would be obvious:

$$
\begin{aligned}
\left.\mathscr{L}_{\tilde{Y}}(\tilde{X}\lrcorner \gamma\right) \mid \Omega & \left.\left.=\lim _{t \rightarrow 0}\{\tilde{X}\lrcorner \gamma-\psi_{-t}^{*}(\tilde{X}\lrcorner \gamma\right)\right\} \mid \Omega \\
& \left.\left.=\lim _{t \rightarrow 0}\{\tilde{X}\lrcorner \gamma \mid \Omega-\psi_{-t}^{*}(\tilde{X}\lrcorner \gamma\right) \mid \Omega\right\} \\
& \left.\left.=\lim _{t \rightarrow 0}\{\tilde{X}\lrcorner \gamma\left|\Omega-\psi_{-t}^{*}(\tilde{X}\lrcorner \gamma\right| \Omega_{-t}\right)\right\}=0
\end{aligned}
$$

the last equality being fulfiled because both $\Omega$ and $\Omega_{-t}$ are states i.e. satisfy Eq. (1.2). In general case $\psi_{t}$ has to be replaced by the group of diffeomorphisms $G_{t}$ generated by $\tilde{Y}$. We must also replace $\Omega_{-t}=\psi_{-t}(\Omega)$ by $G_{-t}(\Omega)$. But it follows from (2.4) that in a neighbourhood of $t=0$ both surfaces $\psi_{-t}(\Omega)$ and $G_{-t}(\Omega)$ "are equal up to terms of second order in the variable $t$ ". It means that (6.5) is satisfied.

We shall now rewrite the Eq. (2.5) in the form which will be useful in later calculations.

We denote $\tilde{Z}_{0}=\tilde{Y}$. Let $\tilde{Z}_{1}, \ldots, \tilde{Z}_{n}$ be vector fields on a neighbourhood of $\Omega$ tangent to $\Omega$ at points belonging to $\Omega$.

Using formulae (6.1), (6.2), and (6.3) we have

$$
\begin{aligned}
& \left.\mathscr{L}_{\tilde{Z}_{0}}(\tilde{X}\lrcorner \gamma\right)\left(\tilde{Z}_{1}, \ldots, \tilde{Z}_{n}\right) \\
& \left.\left.\left.\left.=\left(\tilde{Z}_{0}\right\lrcorner d(\tilde{X}\lrcorner \gamma\right)\right)\left(\tilde{Z}_{1}, \ldots, \tilde{Z}_{n}\right)+d\left(\tilde{Z}_{0}\right\lrcorner \tilde{X}\right\lrcorner \gamma\right)\left(\tilde{Z}_{1}, \ldots, \tilde{Z}_{n}\right) \\
& =\sum_{k=0}^{n}(-1)^{k} \tilde{Z}_{k}\left((\tilde{X} \downarrow \gamma)\left(\tilde{Z}_{0}, \ldots \ldots, \tilde{Z}_{n}\right)\right) \\
& \left.+\sum_{0 \leqq k<s \leqq n}(-1)^{k+s}(\tilde{X}\rfloor \gamma\right)\left(\left[\tilde{Z}_{k}, \tilde{Z}_{s}\right], \tilde{Z}_{0}, \ldots \ldots \ldots, \tilde{Z}_{n}\right) \\
& \left.\left.+\sum_{k=1}^{n}(-1)^{k+1} \tilde{Z}_{k}\left(\left(\tilde{Z}_{0}\right\rfloor \tilde{X}\right\rfloor \gamma\right)\left(\tilde{Z}_{1}, \ldots, \ldots, \tilde{Z}_{n}\right)\right) \\
& \left.+\sum_{1 \leqq k<s \leqq n}(-1)^{k+s}\left(\tilde{Z}_{0}\right\rfloor \tilde{X} \downarrow \gamma\right)\left(\left[\tilde{Z}_{k}, \tilde{Z}_{s}\right], \tilde{Z}_{1}, \ldots \ldots \ldots, \tilde{Z}_{n}\right) \\
& \left.=\tilde{Z}_{0}((\tilde{X}\lrcorner \gamma)\left(\tilde{Z}_{1}, \ldots, \tilde{Z}_{n}\right)\right) \\
& \left.+\sum_{k=1}^{n}(-1)^{k}(\tilde{X}\rfloor \gamma\right)\left(\left[\tilde{Z}_{0}, \tilde{Z}_{k}\right], \tilde{Z}_{1}, \ldots \ldots, \tilde{Z}_{n}\right) \text {. }
\end{aligned}
$$


Now Eq. (2.5) are equivalent the following condition:

For every vector field $\tilde{X}$ given in a neighbourhood of $\Omega$ in $\mathscr{P}$ and arbitrary vector fields $\tilde{Z}_{1}, \ldots, \tilde{Z}_{n}$ given in this neighbourhood and tangent to $\Omega$ on $\Omega$ we have

$$
\left.\left\{\tilde{Y}\left((\tilde{X} \downarrow \gamma)\left(\tilde{Z}_{1}, \ldots, \tilde{Z}_{n}\right)\right)+\sum_{k=1}^{n}(-1)^{k}(\tilde{X}\lrcorner \gamma\right)\left(\left[\tilde{Y}, \tilde{Z}_{k}\right], \tilde{Z}_{1}, \ldots, \ldots, \tilde{Z}_{n}\right)\right\} \mid \Omega=0 .
$$

Remark. The condition (6.7) does not depend on the choise of extension $\tilde{Y}$ of $Y$ onto a neighbourhood of $\Omega$ in $\mathscr{P}$. This fact can be easily seen if we use field Eq. (1.2) written in the form

$$
(\tilde{X} \sqsupset \gamma)\left(\tilde{Z}_{1}, \ldots, \tilde{Z}_{n}\right) \mid \Omega=0
$$

and compute (6.7) in local coordinates on $\Omega$. The formula (6.7) represents a system of linear differential equations for $Y$ which involves only differentiations of $Y$ in directions tangent to $\Omega$. Therefore (6.7) is a differential equation on the submanifold $\Omega$. It follows from (6.8) that if $Y$ is tangent to $\Omega$ then Eq. (6.7) are automatically fulfilled.

Proof of Lemma 1. Let $Y_{1}, Y_{2}$ be vector fields on $\Omega$ and $\tilde{Y}_{1}, \tilde{Y}_{2}$ be any extensions of $Y_{1}, Y_{2}$ onto some neighbourhood of $\Omega$. Fields $\tilde{Y}_{1}$ and $\tilde{Y}_{2}$ fulfil Eq. (6.7).

Using formulae (6.1) and (6.2) we have

$$
\begin{aligned}
d\left(\tilde{Y}_{1} \wedge\right. & \left.\left.\left.\left.\tilde{Y}_{2}\right\lrcorner \gamma\right)\left(\tilde{Z}_{1}, \ldots, \tilde{Z}_{n}\right)=d\left(\tilde{Y}_{2}\right\lrcorner \tilde{Y}_{1}\right\lrcorner \gamma\right)\left(\tilde{Z}_{1}, \ldots, \tilde{Z}_{n}\right) \\
= & (n+1) \sum_{k=1}^{n}(-1)^{k+1} \tilde{Z}_{k}\left(\gamma\left(\tilde{Y}_{1}, \tilde{Y}_{2}, \tilde{Z}_{1}, \ldots \ldots, \tilde{Z}_{n}\right)\right) \\
& +(n+1) \sum_{1 \leqq k<s \leqq n}(-1)^{k+s} \gamma\left(\left[\tilde{Z}_{k}, \tilde{Z}_{s}\right], \tilde{Y}_{1}, \tilde{Y}_{2}, \tilde{Z}_{1}, \ldots \ldots \ldots, \tilde{Z}_{n}\right) .
\end{aligned}
$$

But $d \gamma=0$ and we have from (6.2)

$$
\begin{aligned}
0= & (n+2) d \gamma\left(\tilde{Y}_{1}, \tilde{Y}_{2}, \tilde{Z}_{1}, \ldots, \tilde{Z}_{n}\right) \\
= & \tilde{Y}_{1}\left(\gamma\left(\tilde{Y}_{2}, \tilde{Z}_{1}, \ldots, \tilde{Z}_{n}\right)\right)-\tilde{Y}_{2}\left(\gamma\left(\tilde{Y}_{1}, \tilde{Z}_{1}, \ldots, \tilde{Z}_{n}\right)\right) \\
& +\sum_{k=1}^{n}(-1)^{k+1} \tilde{Z}_{k}\left(\gamma\left(\tilde{Y}_{1}, \tilde{Y}_{2}, \tilde{Z}_{1}, \ldots \ldots, \tilde{Z}_{n}\right)\right)-\gamma\left(\left[\tilde{Y}_{1}, \tilde{Y}_{2}\right], \tilde{Z}_{1}, \ldots, \tilde{Z}_{n}\right) \\
& +\sum_{k=1}^{n}(-1)^{k+1} \gamma\left(\left[\tilde{Y}_{1}, \tilde{Z}_{k}\right], \tilde{Y}_{2}, \tilde{Z}_{1}, \ldots \ldots, \tilde{Z}_{n}\right) \\
& +\sum_{k=1}^{n}(-1)^{k} \gamma\left(\left[\tilde{Y}_{2}, \tilde{Z}_{k}\right], \tilde{Y}_{1}, \tilde{Z}_{1}, \ldots \ldots, \tilde{Z}_{n}\right) \\
& +\sum_{1 \leqq k<s \leqq n}(-1)^{k+s} \gamma\left(\left[\tilde{Z}_{k}, \tilde{Z}_{s}\right], \tilde{Y}_{1}, \tilde{Y}_{2}, \tilde{Z}_{1}, \ldots \ldots \hat{k}_{\hat{s}} \ldots, \tilde{Z}_{n}\right) .
\end{aligned}
$$


If we restrict both sides of (6.10) to the submanifold $\Omega$, use (6.7), (6.8), and (6.9) we obtain at last:

$$
\left.d\left(\left(\tilde{Y}_{1} \wedge \tilde{Y}_{2}\right)\right\lrcorner \gamma\right)\left(\tilde{Z}_{1}, \ldots, \tilde{Z}_{n}\right) \mid \Omega=0 .
$$

Our Theorem 2 follows from the following lemma:

Lemma 2. If $\tilde{Y}_{1}, \tilde{Y}_{2}, \tilde{Y}_{3}$ are vector fields on a manifold $\mathscr{P}$ and $\gamma$ is a closed $(n+1)$-form on $\mathscr{P}(n \geqq 2)$ then:

$$
\begin{aligned}
& \mathscr{L}_{\tilde{Y}_{1}}\left(\tilde{Y}_{3} \downarrow \tilde{Y}_{2} \downarrow \gamma\right)-\mathscr{L}_{\tilde{Y}_{2}}\left(\tilde{Y}_{3} \downarrow \tilde{Y}_{1} \downarrow \gamma\right)+\mathscr{L}_{\tilde{Y}_{3}}\left(\tilde{Y}_{2} \downarrow \tilde{Y}_{1} \downarrow \gamma\right) \\
& \left.\left.\left.\left.\left.\left.-\tilde{Y}_{3}\right\rfloor\left[\tilde{Y}_{1}, \tilde{Y}_{2}\right]\right\lrcorner \gamma+\tilde{Y}_{2}\right\rfloor\left[\tilde{Y}_{1}, \tilde{Y}_{3}\right]\right\lrcorner \gamma-\tilde{Y}_{1}\right\rfloor\left[\tilde{Y}_{2}, \tilde{Y}_{3}\right]\right\lrcorner \gamma \\
& \left.\left.\left.=d\left(\tilde{Y}_{3}\right\lrcorner \tilde{Y}_{2}\right\lrcorner \tilde{Y}_{1}\right\lrcorner \gamma\right) \text {. }
\end{aligned}
$$

Proof of Lemma 2. It follows from (6.3) and (6.4) that

$$
\begin{aligned}
& \left.\left.\left.\left.\left.\left.\mathscr{L}_{\tilde{Y}_{1}}\left(\tilde{Y}_{3}\right\lrcorner \tilde{Y}_{2}\right\lrcorner \gamma\right)=\left[\tilde{Y}_{1}, \tilde{Y}_{3}\right]\right\lrcorner \tilde{Y}_{2}\right\lrcorner \gamma+\tilde{Y}_{3}\right\lrcorner\left[\tilde{Y}_{1}, \tilde{Y}_{2}\right]\right\lrcorner \gamma \\
& \left.\left.\left.+\tilde{Y}_{3}\right\lrcorner \tilde{Y}_{2}\right\lrcorner d\left(\tilde{Y}_{1}\right\lrcorner \gamma\right) .
\end{aligned}
$$

Applying (6.12) we obtain that the left hand side of (6.11) is equal to:

$$
\begin{aligned}
& \left.\left.\left.\left.\left.\left.L=\tilde{Y}_{3}\right\lrcorner\left[\tilde{Y}_{1}, \tilde{Y}_{2}\right]\right\lrcorner \gamma+\tilde{Y}_{2}\right\lrcorner\left[\tilde{Y}_{3}, \tilde{Y}_{1}\right]\right\lrcorner \gamma+\tilde{Y}_{1}\right\lrcorner\left[\tilde{Y}_{2}, \tilde{Y}_{3}\right]\right\lrcorner \gamma \\
& \left.\left.\left.\left.\left.\left.\left.\left.\left.+\tilde{Y}_{3}\right\lrcorner \tilde{Y}_{2}\right\lrcorner d\left(\tilde{Y}_{1}\right\lrcorner \gamma\right)+\tilde{Y}_{1}\right\lrcorner \tilde{Y}_{3}\right\lrcorner d\left(\tilde{Y}_{2}\right\rfloor \gamma\right)+\tilde{Y}_{2}\right\lrcorner \tilde{Y}_{1}\right\lrcorner d\left(\tilde{Y}_{3}\right\lrcorner \gamma\right) .
\end{aligned}
$$

But we have:

$$
\begin{aligned}
&\left.\left.\left.(n(n+1))^{-1}\left(\tilde{Y}_{3}\right\rfloor \tilde{Y}_{2}\right\rfloor d\left(\tilde{Y}_{1}\right\rfloor \gamma\right)\right)\left(\tilde{Z}_{1}, \ldots, \tilde{Z}_{n-1}\right) \\
&=\left.d\left(\tilde{Y}_{1}\right\rfloor \gamma\right)\left(\tilde{Y}_{2}, \tilde{Y}_{3}, \tilde{Z}_{1}, \ldots, \tilde{Z}_{n-1}\right)=\tilde{Y}_{2}\left(\gamma\left(\tilde{Y}_{1}, \tilde{Y}_{3}, \tilde{Z}_{1}, \ldots, \tilde{Z}_{n-1}\right)\right) \\
&-\tilde{Y}_{3}\left(\gamma\left(\tilde{Y}_{1}, \tilde{Y}_{2}, \tilde{Z}_{1}, \ldots, \tilde{Z}_{n-1}\right)\right)+\sum_{k=1}^{n-1}(-1)^{k+1} \tilde{Z}_{k}\left(\gamma\left(\tilde{Y}_{1}, \tilde{Y}_{2}, \tilde{Y}_{3}, \tilde{Z}_{1}, \ldots \ldots, \tilde{Z}_{n-1}\right)\right) \\
&-\gamma\left(\tilde{Y}_{1},\left[\tilde{Y}_{2}, \tilde{Y}_{3}\right], \tilde{Z}_{1}, \ldots, \tilde{Z}_{n-1}\right) \\
&+\sum_{k=1}^{n-1}(-1)^{k+1} \gamma\left(\tilde{Y}_{1},\left[\tilde{Y}_{2}, \tilde{Z}_{k}\right], \tilde{Y}_{3}, \tilde{Z}_{1}, \ldots \ldots, \tilde{Z}_{n-1}\right) \\
&-\sum_{k=1}^{n-1}(-1)^{k+1} \gamma\left(\tilde{Y}_{1},\left[\tilde{Y}_{3}, \tilde{Z}_{k}\right], \tilde{Y}_{2}, \tilde{Z}_{1}, \ldots \ldots, \tilde{Z}_{n-1}\right) \\
&+\sum_{1 \leqq k<s \leqq n-1}(-1)^{k+s} \gamma\left(\tilde{Y}_{1},\left[\tilde{Z}_{k}, \tilde{Z}_{s}\right], \tilde{Y}_{2}, \tilde{Y}_{3}, \tilde{Z}_{1}, \ldots \ldots \ldots, \tilde{Z}_{n-1}\right) .
\end{aligned}
$$

Using equality $d \gamma=0$ we obtain

$$
\begin{aligned}
&\left.\left.\left.(n(n+1))^{-1}\left(\tilde{Y}_{3}\right\rfloor \tilde{Y}_{2}\right\rfloor d\left(\tilde{Y}_{1}\right\lrcorner \gamma\right)\right)\left(\tilde{Z}_{1}, \ldots, \tilde{Z}_{n-1}\right) \\
&= \tilde{Y}_{1}\left(\gamma\left(\tilde{Y}_{2}, \tilde{Y}_{3}, \tilde{Z}_{1}, \ldots, \tilde{Z}_{n-1}\right)\right)-\gamma\left(\left[\tilde{Y}_{1}, \tilde{Y}_{2}\right], \tilde{Y}_{3}, \tilde{Z}_{1}, \ldots, \tilde{Z}_{n-1}\right) \\
&+\gamma\left(\left[\tilde{Y}_{1}, \tilde{Y}_{3}\right], \tilde{Y}_{2}, \tilde{Z}_{1}, \ldots, \tilde{Z}_{n-1}\right) \\
&+\sum_{k=1}^{n-1}(-1)^{k} \gamma\left(\left[\tilde{Y}_{1}, \tilde{Z}_{k}\right], \tilde{Y}_{2}, \tilde{Y}_{3}, \tilde{Z}_{1}, \ldots \ldots, \tilde{Z}_{n-1}\right) .
\end{aligned}
$$


On the other hand we have:

$$
\begin{aligned}
& \left.\left.\left.(n(n+1))^{-1} d\left(\tilde{Y}_{3}\right\lrcorner \tilde{Y}_{2}\right\lrcorner \tilde{Y}_{1}\right\lrcorner \gamma\right)\left(\tilde{Z}_{1}, \ldots, \tilde{Z}_{n-1}\right) \\
& \quad=\sum_{k=1}^{n-1}(-1)^{k+1} \tilde{Z}_{k}\left(\gamma\left(\tilde{Y}_{1}, \tilde{Y}_{2}, \tilde{Y}_{3}, \tilde{Z}_{1}, \ldots \ldots, \tilde{Z}_{n-1}\right)\right) \\
& \quad+\sum_{1 \leqq k<s \leqq n-1}(-1)^{k+s} \gamma\left(\tilde{Y}_{1}, \tilde{Y}_{2}, \tilde{Y}_{3},\left[\tilde{Z}_{k}, \tilde{Z}_{s}\right], \tilde{Z}_{1}, \ldots \ldots \ldots, \tilde{Z}_{n-1}\right) .
\end{aligned}
$$

Using again the condition $d \gamma=0$ and (6.16) we obtain

$$
\begin{aligned}
&\left.\left.\left.(n(n+1))^{-1} d\left(\tilde{Y}_{3}\right\rfloor \tilde{Y}_{2}\right\rfloor \tilde{Y}_{1}\right\rfloor \gamma\right)\left(\tilde{Z}_{1}, \ldots, \tilde{Z}_{n-1}\right) \\
&= \tilde{Y}_{1}\left(\gamma\left(\tilde{Y}_{2}, \tilde{Y}_{3}, \tilde{Z}_{1}, \ldots, \tilde{Z}_{n-1}\right)\right)-\tilde{Y}_{2}\left(\gamma\left(\tilde{Y}_{1}, \tilde{Y}_{3}, \tilde{Z}_{1}, \ldots, \tilde{Z}_{n-1}\right)\right) \\
&+\tilde{Y}_{3}\left(\gamma\left(\tilde{Y}_{1}, \tilde{Y}_{2}, \tilde{Z}_{1}, \ldots, \tilde{Z}_{n-1}\right)\right)-\gamma\left(\left[\tilde{Y}_{1}, \tilde{Y}_{2}\right], \tilde{Y}_{3}, \tilde{Z}_{1}, \ldots, \tilde{Z}_{n-1}\right) \\
&+\gamma\left(\left[\tilde{Y}_{1}, \tilde{Y}_{3}\right], \tilde{Y}_{2}, \tilde{Z}_{1}, \ldots, \tilde{Z}_{n-1}-\gamma\left(\left[\tilde{Y}_{2}, \tilde{Y}_{3}\right], \tilde{Y}_{1}, \tilde{Z}_{1}, \ldots, \tilde{Z}_{n-1}\right)\right. \\
&+\sum_{k=1}^{n-1}(-1)^{k} \gamma\left(\left[\tilde{Y}_{1}, \tilde{Z}_{k}\right], \tilde{Y}_{2}, \tilde{Y}_{3}, \tilde{Z}_{1}, \ldots \ldots, \tilde{Z}_{n-1}\right) \\
& \quad-\sum_{k=1}^{n-1}(-1)^{k} \gamma\left(\left[\tilde{Y}_{2}, \tilde{Z}_{k}\right], \tilde{Y}_{1}, \tilde{Y}_{3}, \tilde{Z}_{1}, \ldots, \ldots, \tilde{Z}_{n-1}\right) \\
&+\sum_{k=1}^{n-1}(-1)^{k} \gamma\left(\left[\tilde{Y}_{3}, \tilde{Z}_{k}\right], \tilde{Y}_{1}, \tilde{Y}_{2}, \tilde{Z}_{1}, \ldots \ldots, \tilde{Z}_{n-1}\right) .
\end{aligned}
$$

If we put (6.15) and its cyclic permutations to (6.13) we obtain (6.17).

Proof of Theorem 2. For $n=1 \gamma$ is a 2-form and the space $\mathscr{H}$ is finite dimensional (a.i.s. are points in $\mathscr{P}$ ). $\mathscr{H}$ is equal to the quotient space $\mathscr{P} / L$ where $L$ is a congruence given by the distribution of singular vectors of $\gamma$. The form $\Gamma$ is the projection of $\gamma$ onto $\mathscr{P} / L$ so it is closed.

Let now $n \geqq 2$ so we can use Lemma 2 . Let $\hat{Y}_{1}, \hat{Y}_{2}, \hat{Y}_{3}$ be vectors tangent to $\mathscr{H}$ at $\Omega$. In order to calculate the value of $d \Gamma_{\Omega}\left(\hat{Y}_{1}, \hat{Y}_{2}, \hat{Y}_{3}\right)$ we must take any vector fields in a neighbourhood of $\Omega$ equal $\hat{Y}_{1}, \hat{Y}_{2}, \hat{Y}_{3}$ at the point $\Omega$ and use the formula (6.2) which reads for $p=2$ :

$$
\begin{aligned}
3 d \Gamma\left(\hat{Y}_{1}, \hat{Y}_{2}, \hat{Y}_{3}\right)= & \hat{Y}_{1} \Gamma\left(\hat{Y}_{2}, \hat{Y}_{3}\right)-\hat{Y}_{2} \Gamma\left(\hat{Y}_{1}, \hat{Y}_{3}\right)+\hat{Y}_{3} \Gamma\left(\hat{Y}_{1}, \hat{Y}_{2}\right) \\
& -\Gamma\left(\left[\hat{Y}_{1}, \hat{Y}_{2}\right], \hat{Y}_{3}\right)+\Gamma\left(\left[\hat{Y}_{1}, \hat{Y}_{3}\right], \hat{Y}_{2}\right)-\Gamma\left(\left[\hat{Y}_{2}, \hat{Y}_{3}\right], \hat{Y}_{1}\right) .
\end{aligned}
$$

Now we can use the possibility of choosing a surface $\varkappa\left(P_{k}\right),\left(\varkappa, P_{k}\right) \in \mathscr{A}$ passing through $\Omega$ and such that fields $\hat{Y}_{i} i=1,2,3$ are tangent to $\varkappa\left(P_{k}\right)$. The mapping $x$ is given by a smooth mapping

$$
P_{k} \times \mathscr{P} \ni(t ; p) \rightarrow \psi(\boldsymbol{t} ; p) \in \mathscr{P},
$$

where $\psi(\boldsymbol{t} ; \cdot)$ are diffeomorphisms of $\mathscr{P}$ and $\psi(0 ; \cdot)=\mathrm{id}$. In the manifold $P_{k} \times \mathscr{P}$ we take a submanifold $\mathscr{K}=\left\{(\boldsymbol{t} ; \psi(\boldsymbol{t} ; p)) \in P_{k} \times \mathscr{P}: \boldsymbol{t} \in P_{k}, p \in \Omega\right\}$. 
We have the sequence of smooth mappings

$$
\mathscr{K} \stackrel{i}{\rightarrow} P_{k} \times \mathscr{P} \stackrel{\pi}{\rightarrow} \mathscr{P},
$$

where $\pi$ is the natural projection and $i$ is the natural embedding.

Take in $\mathscr{K}$ the $(n+1)$-form $\tilde{\gamma}$ which is the pull-back of $\gamma$ from $\mathscr{P}$ onto $\mathscr{K}$ :

$$
\tilde{\gamma}=i^{*} \pi^{*} \gamma \text {. }
$$

Given any diffeomorphism $\varphi: P_{k} \rightarrow P_{k}$ there is the canonical lift of $\varphi$ to $\mathscr{K}$ :

$$
\tilde{\varphi}(\boldsymbol{t}, \psi(\boldsymbol{t} ; p))=(\varphi(\boldsymbol{t}), \psi(\varphi(\boldsymbol{t}) ; p)) \text {. }
$$

It is easy to see that $\tilde{\varphi}$ is a diffeomorphism: $\mathscr{K} \rightarrow \mathscr{K}$.

Our lift preserves group properties:

$$
\left(\varphi_{1} \circ \varphi_{2}\right)^{-}=\tilde{\varphi}_{1} \circ \tilde{\varphi}_{2} ; \quad\left(\varphi^{-1}\right)^{\sim}=\tilde{\varphi}^{-1}
$$

This allows us do define the lift of a vector field in $P_{k}$ to $\mathscr{K}$.

The generator of a local 1-parameter group of diffeomorphisms $\varphi_{\tau}$ is namely lifted to the generator of $\tilde{\varphi}_{\tau}$. It follows from (6.21) that this lift preserves Lie bracket:

$$
\left[Y_{1}, Y_{2}\right]=\left[\tilde{Y}_{1}, \tilde{Y}_{2}\right]
$$

It is easy to check that

$$
\left.\left.\Gamma_{\varkappa(t)}(\hat{X}, \hat{Y})=\int_{c_{t}}(X \wedge Y)\right\lrcorner \gamma=\int_{\tilde{c}_{t}}(\tilde{X} \wedge \tilde{Y})\right\lrcorner \tilde{\gamma},
$$

where $\tilde{c}_{\boldsymbol{t}}=\left\{(\boldsymbol{t}, q): q \in c_{\boldsymbol{t}}\right\}: c_{\boldsymbol{t}} \in \mathscr{C}$ and $c_{\boldsymbol{t}} \subset \chi(\boldsymbol{t})$.

Because $c_{\boldsymbol{t}}$ is arbitrary we can take $c_{\boldsymbol{t}}=\{\psi(\boldsymbol{t} ; p): p \in c\}$, where $c$ is any a.i.s. contained in $\Omega=x(0)$.

Now if $\hat{Z}$ is a vector field tangent to $\chi\left(P_{k}\right)$ and $\varphi_{\tau}$ is a local 1-parameter group of diffeomorphisms generated in $P_{k}$ by pull-back of $\hat{Z}$ then:

$$
\begin{aligned}
& \left.\left.[\hat{Z} \Gamma(\hat{X}, \hat{Y})](\chi(\boldsymbol{t}))=\lim _{\tau \rightarrow 0}\left\{\int_{\tilde{\varphi}_{\tau}\left(\tilde{c}_{t}\right)}(\tilde{X} \wedge \tilde{Y})\right\rfloor \tilde{\gamma}-\int_{\tilde{c}_{t}}(\tilde{X} \wedge \tilde{Y})\right\rfloor \tilde{\gamma}\right\} \\
& \left.\left.\left.\quad=\lim _{\tau \rightarrow 0} \int_{\tilde{c}_{t}}\left[\tilde{\varphi}_{\tau}^{*}(\tilde{X} \wedge \tilde{Y})\right\rfloor \tilde{\gamma}-(\hat{X} \wedge \hat{Y})\right\rfloor \tilde{\gamma}\right]=\int_{\tilde{c}_{t}} \mathscr{L}_{\tilde{Z}}[(\tilde{X} \wedge \tilde{Y})\rfloor \tilde{\gamma}\right] .
\end{aligned}
$$

We can change the order of integrating and passing to the limit because everything is smooth and supports of forms are compact.

Using (6.22), (6.23), and (6.24) we can rewrite the left hand side of (6.18) in the following way:

$$
3 d \Gamma\left(\hat{Y}_{1}, \hat{Y}_{2}, \hat{Y}_{3}\right)=\int_{\tilde{c}} d \alpha,
$$

where $d \alpha$ is equal to left hand side of (6.11). Using formula (6.11) we see that $\left.\left.\left.\alpha=\tilde{Y}_{3}\right\lrcorner \tilde{Y}_{2}\right\lrcorner \tilde{Y}_{1}\right\lrcorner \tilde{\gamma}$. The form $\alpha$ has a compact support therefore

$$
\int_{\tilde{c}} d \alpha=0 \text {. }
$$


Proof of Theorem 3. Let $F_{1}, F_{2}$ be physical quantities $\hat{Z}, \hat{Z}_{1}, \hat{Z}_{2}$-vector fields on $\mathscr{H}$ and

$$
d F_{k} \cdot \hat{Z}=\left\langle\hat{Z},-\hat{Y}_{k}^{b}\right\rangle=\Gamma\left(\hat{Z}, \hat{Y}_{k}\right) \quad k=1,2
$$

We have:

$$
\begin{aligned}
0 & =2 d^{2} F_{k}\left(\hat{Z}_{1}, \hat{Z}_{2}\right) \\
& =\hat{Z}_{1}\left(d F_{k} \cdot \hat{Z}_{2}\right)-\hat{Z}_{2}\left(d F_{k} \hat{Z}_{1}\right)-d F_{k}\left(\left[\hat{Z}_{1}, \hat{Z}_{2}\right]\right) .
\end{aligned}
$$

From (6.25) and (6.26) we have for $k=1 \hat{Z}_{1}=\hat{Z}, \hat{Z}_{2}=\hat{Y}_{2}$

$$
\hat{Z}\left(\Gamma\left(\hat{Y}_{2}, \hat{Y}_{1}\right)=\hat{Y}_{2}\left(\Gamma\left(\hat{Z}, \hat{Y}_{1}\right)\right)+\Gamma\left(\left[\hat{Z}, \hat{Y}_{2}\right], \hat{Y}_{1}\right)\right.
$$

and for $k=2 \hat{Z}_{1}=\hat{Z}, \hat{Z}_{2}=\hat{Y}_{1}$

$$
\hat{Z}\left(\Gamma\left(\hat{Y}_{1}, \hat{Y}_{2}\right)\right)=\hat{Y}_{1}\left(\Gamma\left(\hat{Z}, \hat{Y}_{2}\right)\right)+\Gamma\left(\left[\hat{Z}, \hat{Y}_{1}\right], \hat{Y}_{2}\right) .
$$

On the other hand we have $d \Gamma=0$ :

$$
\begin{aligned}
0= & 3 d \Gamma\left(\hat{Z}, \hat{Y}_{1}, \hat{Y}_{2}\right)=\hat{Z}\left(\Gamma\left(\hat{Y}_{1}, \hat{Y}_{2}\right)\right)-\hat{Y}_{1}\left(\Gamma\left(\hat{Z}, \hat{Y}_{2}\right)\right)+\hat{Y}_{2}\left(\Gamma\left(\hat{Z}, \hat{Y}_{1}\right)\right) \\
& -\Gamma\left(\left[\hat{Z}, \hat{Y}_{1}\right], \hat{Y}_{2}\right)+\Gamma\left(\left[\hat{Z}, \hat{Y}_{2}\right], \hat{Y}_{1}\right)-\Gamma\left(\left[\hat{Y}_{1}, \hat{Y}_{2}\right], \hat{Z}\right) .
\end{aligned}
$$

From (6.27), (6.28), and (6.29) we obtain

$$
d\left\{F_{1}, F_{2}\right\} \hat{Z}=\hat{Z}\left(\Gamma\left(\hat{Y}_{1}, \hat{Y}_{2}\right)\right)=\Gamma\left(\hat{Z},\left[\hat{Y}_{1}, \hat{Y}_{2}\right]\right),
$$

so we have

$$
d\left\{F_{1}, F_{2}\right\}=-\left[\hat{Y}_{1}, \hat{Y}_{2}\right]^{b} .
$$

Proof of Proposition 1. From Theorem 3 we have:

$$
\begin{aligned}
J & =\left\{\left\{F_{1}, F_{2}\right\}, F_{3}\right\}+\left\{\left\{F_{2}, F_{3}\right\}, F_{1}\right\}+\left\{\left\{F_{3}, F_{1}\right\}, F_{2}\right\} \\
& =\Gamma\left(\left[\hat{Y}_{1}, \hat{Y}_{2}\right], \hat{Y}_{3}\right)+\Gamma\left(\left[\hat{Y}_{2}, \hat{Y}_{3}\right], \hat{Y}_{1}\right)+\Gamma\left(\left[\hat{Y}_{3}, \hat{Y}_{1}\right], \hat{Y}_{2}\right) .
\end{aligned}
$$

Using the formula (6.30) we have

$$
J=-\hat{Y}_{3} \Gamma\left(\hat{Y}_{1}, \hat{Y}_{2}\right)-\hat{Y}_{1} \Gamma\left(\hat{Y}_{2}, \hat{Y}_{3}\right)-\hat{Y}_{2} \Gamma\left(\hat{Y}_{3}, \hat{Y}_{1}\right) .
$$

If we add (6.32) and (6.33) we obtain minus right hand side of (6.29) where $\hat{Z}$ is replaced by $\hat{Y}_{3}$. Thus $2 J=0$.

The proof of Proposition 2 follows immediately from the formula (6.18) and Theorem 2. If $\hat{Y}_{1}, \hat{Y}_{2} \in W$ then (6.18) reads:

$0=3 d \Gamma\left(\hat{Y}_{1}, \hat{Y}_{2}, \hat{Y}_{3}\right)=-\Gamma\left(\left[\hat{Y}_{1}, \hat{Y}_{2}\right], \hat{Y}_{3}\right)$ for any field $\hat{Y}_{3}$. It means that $\left[\hat{Y}_{1}, \hat{Y}_{2}\right] \in W$.

\section{References}

1. Bałaban, T., Rączka, R.: Second quantization of non-linear relativistic wave equations, Part I: Canonical formalism (preprint)

2. Białynicki-Birula, I.: Nuovo Cimento 35, 697 (1965)

3. Białynicki-Birula,I., Iwiński, Z.: Reports Math. Phys. 4, 139 (1973)

4. Bourbaki, N.: Topologie générale. Paris: 1960

5. Dedecker,P.: Calcul des variations, formes différentielles et champs géodésiques. Colloque International de Géometrie Différentielle. Strassbourg: 1953 